\title{
Diagnosis of Lymphocytic Thyroiditis Based on Doppler Findings Validated Against Fine Needle Aspiration Cytology
}

\author{
Authors \\ Amilu Elsa Varghese ${ }^{1}$, Reeba George Pulinilkunnathil ${ }^{2}$, Beenamol S ${ }^{3}$, N Roy ${ }^{4}$, \\ Harikumaran Nair ${ }^{5}$ \\ ${ }^{1}$ Formerly Junior Resident, Dept of Radiodiagnosis Govt TD Medical College Alappuzha, Kerala India \\ Email: amiluelsa@gmail.com \\ ${ }^{2}$ Assistant Professor, Dept of Radiodiagnosis, Govt Medical College Thiruvananthapuram, Kerala India \\ ${ }^{3}$ Associate Professor, Dept of Radiodiagnosis, Govt Medical College Thiruvananthapuram, Kerala India \\ ${ }^{4}$ Professor and Head of Department, Dept of Radiodiagnosis, Govt Medical College, Thiruvananthapuram, \\ Kerala, India \\ ${ }^{5}$ Professor and Head of Department, Dept of Radiodiagnosis, Govt TD Medical College, Alappuzha, Kerala, \\ India \\ Corresponding Author \\ Reeba George Pulinilkunnathil \\ Assistant Professor, Dept of Radiodiagnosis, Govt Medical College Thiruvananthapuram, Kerala India \\ Email:reebageorge68@gmail.com \\ Abstract \\ Background: Lymphocytic thyroiditis is the most common form of subclinical and overt hypothyroidism in \\ an iodine sufficient region. It is the present manifestation of thyroid diseases in the post iodization era. The \\ aim of this study is to validate the Doppler findings in lymphocytic thyroiditis \\ Materials and Methods: Diagnostic test evaluation was done by comparing the Doppler findings with fine \\ needle aspiration cytology which is taken as the reference standard. 89 patients with diffuse neck swelling in \\ the age group 18-80 years, who came for sonographic examination of the thyroid to the Department of \\ Radiodiagnosis, Government TD Medical college Alappuzha were assessed. \\ Result: The Doppler findings in the study were classified into three patterns and found that $68 \%$ of patients \\ with pattern 2 and pattern 3 had lymphocytic thyroiditis. A new cut off value for Resistive Index was \\ calculated by plotting. Receiver operating Characteristic curve. \\ Conclusion: The sensitivity, specificity, PPV and NPV of increased vascularity (pattern 2\&3) in diagnosing \\ lymphocytic thyroiditis was found to be $71 \%, 52 \%, 80 \%$ and $48 \%$ respectively. Diagnosing lymphocytic \\ thyroiditis by pattern 3 vascularity had a high specificity and positive predictive value of 88 and 91 \\ respectively. A new cut off value of RI was identified as 0.61 with a sensitivity of $78 \%$ and specificity of $65 \%$ \\ from the ROC curve. \\ Keywords-Colour Doppler, Resistive index, Lymphocytic Thyroiditis, Spectral pattern.
}




\section{Introduction}

Lymphocytic thyroiditis, is an autoimmune inflamematory disease caused by activation of CD4 helper T- lymphocytes specific for thyroid antigen and characterized by lymphocyte infiltration, fibrosis, and gradual destruction of the thyroid gland. The study aims at validating the Doppler findings in lymphocytic thyroiditis.

Lymphocytic thyroiditis is the most common form of thyroiditis in Kerala. Moreover there are few studies regarding the Doppler findings of lymphocytic thyroiditis in Kerala.

\section{Diagnosis of Lymphocytic Thyroiditis-}

Clinically, patients usually present with thyroid enlargement and symptoms of hypothyroidism. The clinical diagnostic criteria for lymphocytic thyroiditis, as per the Japan Thyroid Association (1) is:

\section{Clinical Findings:-}

a) Diffuse swelling of the gland without any other cause

\section{Laboratory Findings:-}

a) Positive for anti-thyroid microsomal antibody or anti-thyroid peroxidase (TPO) antibody.

b) Positive for anti-thyroglobulin antibody.

c) Lymphocytic infiltration in the thyroid gland confirmed with cytological examination.

\section{Criteria}

A patient shall be said to have chronic thyroiditis if he/she has satisfied clinical criterion and any one laboratory criterion (1)

The sonological diagnosis of lymphocytic thyroiditis can be considered when the thyroid parenchyma appears coarsened and hypoechoic. The hypo-echogenicity is attributed to lymphocyte infiltration and disruption of architecture

The end stage thyroiditis can be diagnosed when the gland is small and atrophic, with reduced vascularity in colour Doppler imaging.

\section{Materials and Method}

The study was designed as a Diagnostic test evaluation conducted in department of Radiodiagnosis, during the period January 2014- June 2015. Patients in the age group 18-80 who came for sonographic evaluation of neck with diffuse neck swelling were included in the study. Patients who did not give consent, and having post-partum thyroiditis were excluded from the study.

\section{a. Study Instrument}

Sonographic examination was done using a Siemens Acuson sonographic machine with VF 13- $5 \mathrm{MHz}$ linear transducer.

\section{b. Study Procedure and Analysis}

The study was done after obtaining ethical clearance from the Human ethical committee of the institution. Patients $(n=89)$ in the age group 18-80 who came for sonographic evaluation of neck with diffuse neck swelling were evaluated initially. The Doppler evaluation was followed by fine needle aspiration cytology. The cytology results were inconclusive for seven patients, so they were excluded from the evaluation. The findings of 82 patients were then analysed and sensitivity, specificity, positive predictive value, negative predictive value was calculated. The Doppler findings in thyroiditis is categorised into three patterns in the present study.

Table 1- The Doppler patterns in lymphocytic thyroiditis.

\section{Pattern 1- absent parenchymal vascularity}

Pattern 2-mild increase in parenchymal flow in uneven distribution

Pattern 3- markedly increased blood flow in diffuse distribution.

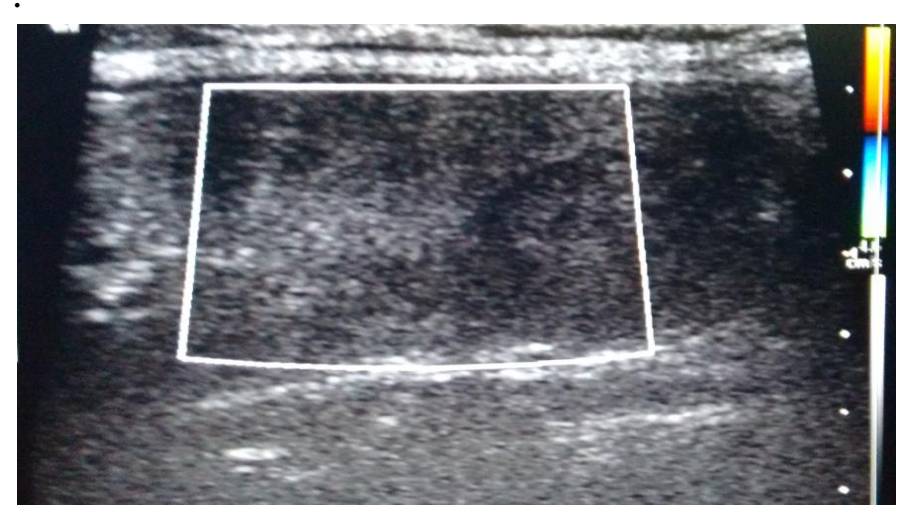

Figure1- Pattern 1 vascularity 


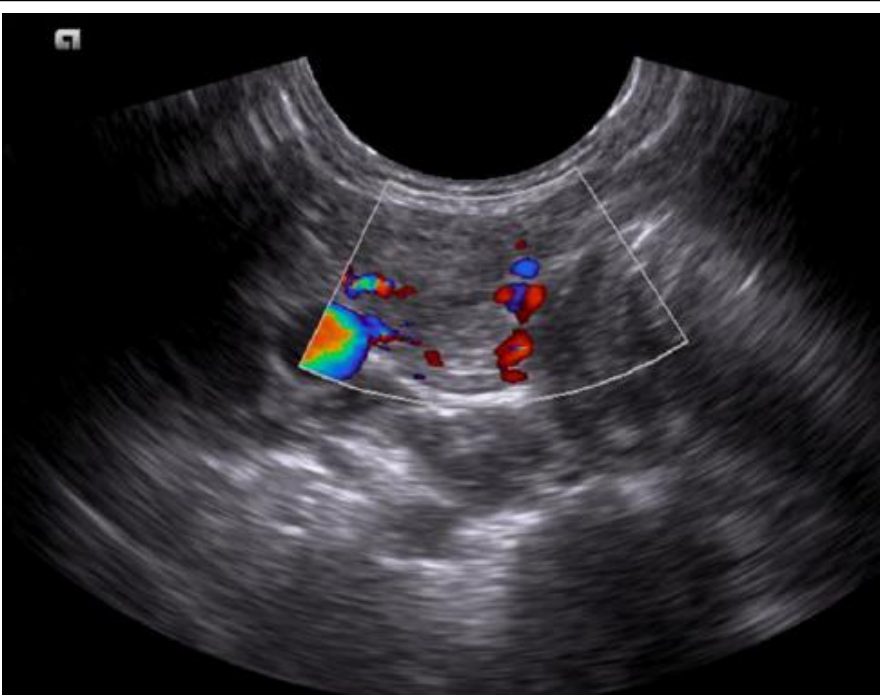

Figure 2- Pattern 2 vascularity

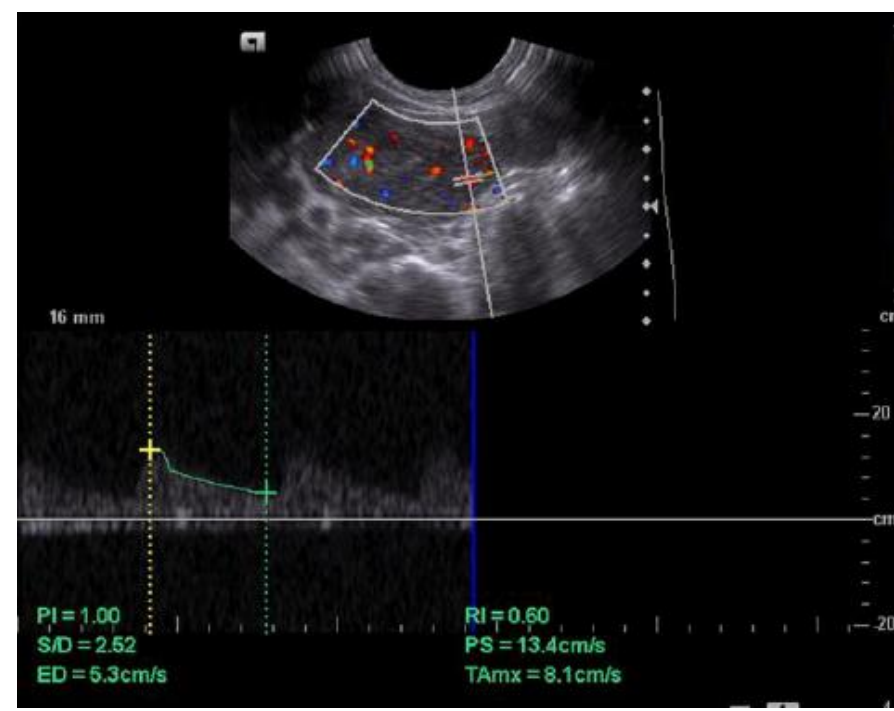

Figure 3- Pattern 3 vascularity

Patterns 2 and 3 were considered as having increased vascularity

RI measurements were performed within each lobe of the thyroid in the inferior or superior thyroid artery. The value obtained for each lobe were averaged for one patient

The R.I value in normal thyroid is $0.7+/-0.05$

\subsection{Statistical Evaluation and Results}

82 patients in the age group 18-80 who came for sonographic evaluation of neck with diffuse neck swelling were available for analysis

And in the study sample, Doppler pattern $2 \& 3$ was seen in the following conditions.
Table 2- showing the increased vascularity (pattern $2 \& 3)$ and cytology results.

\begin{tabular}{|l|l|c|c|c|c|c|}
\hline \multicolumn{2}{|l|}{} & Normal & $\begin{array}{c}\text { Nodular } \\
\text { thyroid } \\
\text { disease }\end{array}$ & $\begin{array}{c}\text { Lymphocytic } \\
\text { thyroiditis }\end{array}$ & $\begin{array}{c}\text { Papillary } \\
\text { carcinoma in } \\
\text { background } \\
\text { of thyroiditis }\end{array}$ & $\begin{array}{c}\text { Medullary } \\
\text { carcinoma }\end{array}$ \\
\hline \multirow{2}{*}{ Vascularity } & Increased & 3 & 8 & 39 & 6 & 1 \\
\cline { 2 - 8 } & Normal & 7 & 5 & 13 & 0 & 0 \\
\hline Total & 10 & 13 & 52 & 6 & 1 \\
\hline
\end{tabular}

However in majority of patients with lymphocytic thyroiditis pattern 3 was found $(51 \%)$

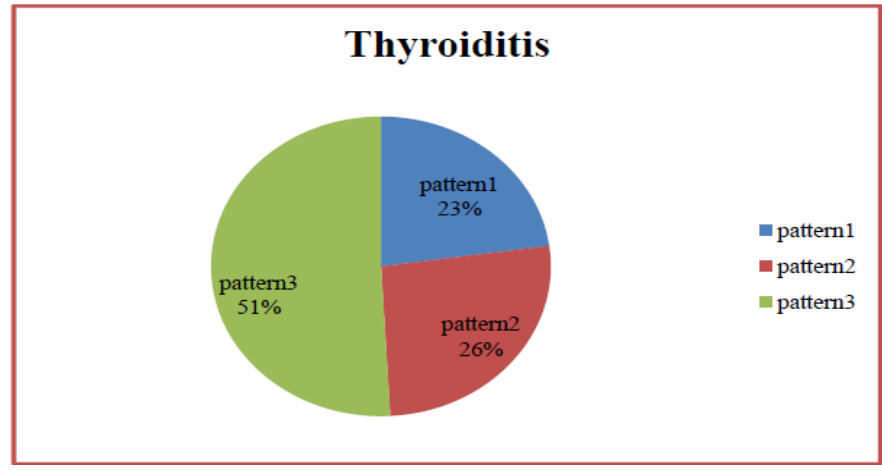

Figure 4- Pie-chart showing the proportion of vascularity patterns in patients diagnosed as lymphocytic thyroiditis.

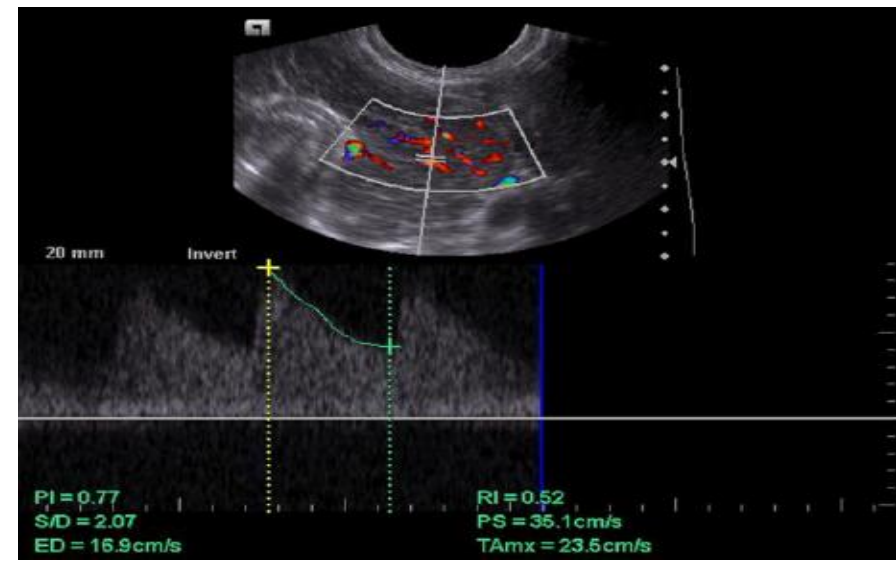

Figure 5-Spectral pattern in lymphocytic thyroiditis showing low velocity pattern

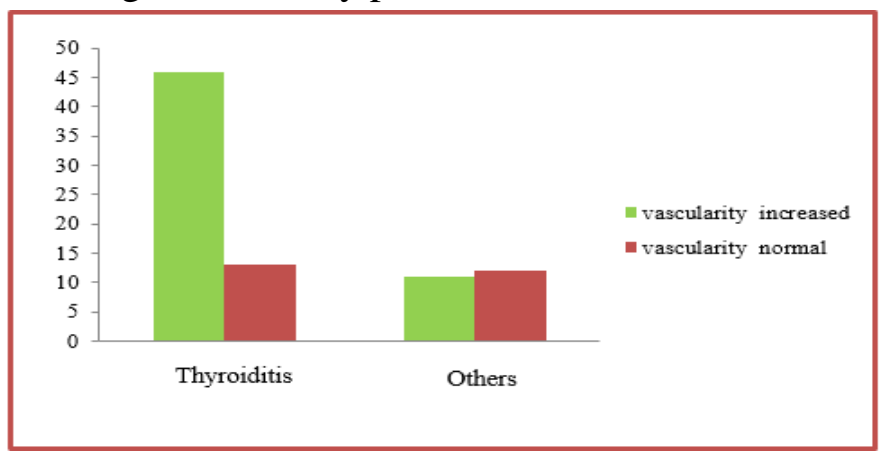

Figure 6-Bar chart showing relationship of increased vascularity (pattern 2\&3) and lymphocytic thyroiditis. 
Both increased and normal vascularity patterns can occur in lymphocytic thyroiditis. But the increased vascularity pattern is seen predominantly in the present study.

The sensitivity, specificity, PPV and NPV of increased vascularity (pattern $2 \& 3$ ) in diagnosing lymphocytic thyroiditis was $71 \%, 52 \%, 80 \%$ and $48 \%$ in the present study. Pattern 3 vascularity was found to have a sensitivity, specificity, PPV, NPV of $51 \%, 88 \%, 91 \%, 44 \%$ in diagnosing lymphocytic thyroiditis.

A wide range of Resistive index values were seen in the patients diagnosed as lymphocytic thyroiditis.

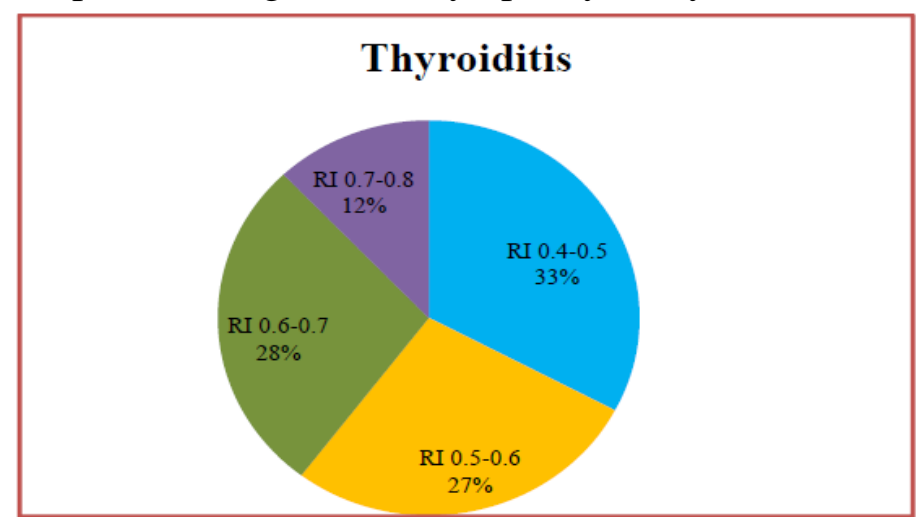

Figure 7- Proportion of range of resistive indices in patients with cytology proven lymphocytic thyroiditis

About $33 \%$ patients diagnosed as lymphocytic thyroiditis have RI value in the range of (0.4-0.5). The sensitivity and specificity for RI value in the range of (0.4-0.5) in diagnosing lymphocytic thyroiditis was found to be $32 \%$ and $86.9 \%$.

The RI values were plotted in a Receiver Operating Characteristic Curve. A new cut off value for RI of 0.61, was identified with a sensitivity and specificity of $71 \%$ and $65 \%$ respectively.

\section{Discussion}

The colour Doppler findings in lymphocytic thyroiditis is increased vascularity. The increase in vascularity is attributed to the trophic effect of TSH in lymphocytic thyroiditis. $77 \%$ of patients with lymphocytic thyroiditis have pattern 2 and 3 vascularity (Figure 4). Pattern 3 was seen in $51 \%$ of lymphocytic thyroiditis in the present study. This is concordance with classification pattern by Schultz et $\mathrm{al}^{2}$.

A comprehensive study by Schultz et $\mathrm{al}^{2}$ established that a positive correlation exist between TSH, TPOAb and TgAb levels and they found that colour intensity was inversely proportional to the duration of hypothyroidsm. Autoimmunity also plays a role in thyroid hyper-vascularity as seen by the studies of Acar et $\mathrm{al}^{3}$. The sensitivity, specificity, PPV and NPV of increased vascularity (pattern $2 \& 3$ ) in diagnosing lymphocytic thyroiditis is $71 \%, 52 \%, 80 \%$ and $48 \%$ in the present study consistent with findings of Schultz et $\mathrm{al}^{2}$, Boggazi etal ${ }^{4}$ and Acar et $\mathrm{al}^{3}$.

The spectral pattern in lymphocytic thyroiditis shows low velocity flow pattern. Hyper-vascularity in Doppler is seen in lymphocytic thyroiditis and Graves disease however both can be differentiated by the peak systolic velocity, as the latter has high flow velocity ${ }^{5}$

A wide range of resistive indices (RI) was seen in the study sample consistent with the findings of Acari et $\mathrm{al}^{3}$ and

Sarikaya et al6 (Figure7). The new cut off value for RI in lymphocytic thyroiditis identified from ROC curve was 0.6 , less than the RI value of normal thyroid parenchyma. This is due to the increased vascularity in lymphocytic thyroiditis.

The parameter having maximum sensitivity for diagnosing Lymphocytic thyroiditis is increased vascularity (Pattern 2\&3) and that with maximum specificity is Pattern ${ }^{3}$.

\section{Conclusions}

Lymphocytic thyroiditis shows hyper-vascularity in Colour Doppler imaging.

Pattern 2 and 3 of vascularity was seen in $77 \%$ of patients with Lymphocytic thyroiditis and have the maximum sensitivity $(71 \%)$ in diagnosing the same.

Pattern 3 was seen in $51 \%$ of lymphocytic thyroiditis in the present study and has the maximum specificity (88\%) in diagnosing the disease, among the Doppler patterns.

A new cut-off value of Resistive index of 0.61 was identified with a sensitivity and specificity of $71 \%$ 
and $65 \%$ respectively in diagnosing lymphocytic thyroiditis.

\section{Acknowledgement}

Our sincere thanks to department of Pathology, Govt .TD Medical College Alappuzha.

\section{References}

1. http://www.japanthyroid.jp/en/guidelines.ht ml. [Online].; 2010 [cited 2015 oct 15] Available from: http://www.japanthyroid.jp/en/guidelines.ht $\mathrm{ml}$.

2. Schulz SL, Uwe S, Jurgen HH. Colour doppler sonography in hypothyroidism Eur $\mathbf{J}$ Ultrasound 2003,16(3): 183-9.

3. Turker Acar, Suha Sureyya Ozbek, Mehmet Erdoğan, Ahmet Gokhan Ozgen, Selçuk Orhan Demirel. US findings in euthyroid patients with positive antithyroid autoantibody tests compared to normal and hypothyroid cases. Turkish society of radiology. 2013; 19:265-270.

4. Bogazzi F, Bartalena L, Brogioni S, Burelli A, Manetti L, Tanda ML, et al.Thyroid vascularity and blood flow are not dependent on serum thyroid hormonelevels: studies in vivo by color flow Doppler sonography. Eur J Endocrinol. 1999;140(5):452-6

5. Carol Rumack, Stepanie R Wilson, J William Charboneau Deborah Levine. diagnostic ultrasound. 4th ed. gaertner r, editor. philadelphia: elsevier; 2011.

6. Basar Sarikaya, I,III Huseyin Demirbilek,II Deniz Akata,I Nurgun Kandemir. The role of the resistive index in Hashimoto's thyroiditis- a sonographic pilot study in children. Clinical science. 2012; 67(11):1253-1257. 